\title{
INDICADORES DE QUALIDADE EM ENFERMAGEM COM ÊNFASE NO CENTRO CIRÚRGICO: REVISÃO INTEGRATIVA DA LITERATURA
}

\author{
Quality indicators in nursing with emphasis in the surgical center: integrative literature review
} Indicadores de calidad en enfermería con énfasis en el quirófano: revisión integrativa de la literatura

\author{
Juliana Aparecida Baldo Amaral', Wilza Carla Spiri², Silvia Cristina Mangini Bocchi
}

RESUMO: Objetivo: Analisar as produções nacional e internacional dos indicadores de qualidade em Enfermagem no Centro Cirúrgico. Método: Revisão integrativa da literatura de 2009 a 2016 de artigos indexados nas bases de dados: Literatura Latino-Americana e do Caribe em Ciências da Saúde, Scientific Electronic Library Online e US National Library of Medicine. A amostra final foi composta por 17 artigos. Resultados: Houve predominância das publicações nacionais, em 2013, realizadas por enfermeiros mestres, com abordagem quantitativa. Os temas dessas foram: importância, confiabilidade e benefícios dos indicadores de qualidade para o gerenciamento da assistência de Enfermagem; principais dificuldades quanto ao uso dos indicadores; sistema informatizado para coleta de dados dos indicadores e visão dos enfermeiros a respeito do uso dessa ferramenta de qualidade. Conclusão: Foram observados os benefícios e as principais dificuldades referentes à utilização dos indicadores de qualidade para o Centro Cirúrgico. No entanto, a avaliação de desempenho do Centro Cirúrgico por meio de indicadores ainda é pouco utilizada. Palavras-chave: Indicadores de qualidade em assistência à saúde. Enfermagem. Centros cirúrgicos. Gerenciamento da prática profissional.

ABSTRACT: Objective: To analyze the national and international scientific work concerning quality indicators in nursing in the surgical center. Method: This is an integrative review of the literature from 2009 to 2016 of articles indexed in the databases Latin American and Caribbean Literature in Health Sciences, Scientific Electronic Library Online, and US National Library of Medicine. The final sample included 17 articles. Results: In 2013, scientific studies carried out by master nurses with a quantitative approach were predominant. The themes of these studies were importance, reliability, and benefits of the quality indicators for the management of nursing care; difficulties in using the indicators; computerized system for data collection of indicators; and nurses' perspective on the use of this tool for the quality assessment. Conclusion: Benefits and main difficulties on the use of quality indicators for the surgical centers were observed. However, the surgical center performance assessment by means of indicators is still poorly used.

Keywords: Quality indicators, health care. Nursing. Surgicenters. Practice management.

RESUMEN: Objetivo: Analizar las producciones nacionales e internacionales de los indicadores de calidad en Enfermería en el Quirófano. Método: Revisión integrativa de la literatura de 2009 a 2016 de artículos indexados en las bases de datos: Literatura Latinoamericana y del Caribe en Ciencias de la Salud, Scientific Electronic Library Online y US National Library of Medicine. La muestra final fue compuesta por 17 artículos. Resultados: Hubo predominancia de las publicaciones nacionales, en 2013, realizadas por enfermeros maestros, con abordaje cuantitativo. Los temas de esas publicaciones fueron: importancia, confiabilidad y beneficios de los indicadores de calidad para la gestión de la asistencia de Enfermería; principales dificultades con relación al uso de los indicadores; sistema informatizado para colecta de datos de los indicadores y visión de los enfermeros al respecto del uso de esa herramienta de calidad. Conclusión: Fueron observados los beneficios y las principales dificultades referentes a la utilización de los indicadores de calidad para el Quirófano. Sin embargo, la evaluación de desempeño del Quirófano por medio de indicadores aún es poco utilizada.

Palabras clave: Indicadores de calidad de la atención de salud. Enfermería. Centros quirúrgicos. Gestión de la práctica profesional.

'Enfermeira, Mestre em Enfermagem pela Universidade Estadual Paulista “Júlio de Mesquita Filho”, Servidora Pública Estadual do Instituto Lauro de Souza Lima-Bauru (SP), Brasil. E-mail: julianaabaldo@hotmail.com ${ }^{2}$ Enfermeira, Doutora em Enfermagem, Professora Adjunta do Departamento de Enfermagem da Universidade Estadual Paulista “Júlio de Mesquita Filho" - Botucatu (SP), Brasil. E-mail: wilza@fmb.unesp.br ${ }^{3}$ Enfermeira, Doutora em Enfermagem, Professora-Assistente Doutora do Departamento de Enfermagem da Universidade Estadual Paulista "Júlio de Mesquita Filho" - Botucatu (SP), Brasil. E-mail: sbocchi@fmb.unesp.br Rua Teizi Tokuhara, 1-120 - Condomínio Quinta Ranieri Green, CEP: 17055-800 - Bauru (SP), Brasil.

Recebido: 17 ago. 2016 - Aprovado: 04 nov. 2016

DOI: $10.5327 / Z 1414-4425201700010008$ 


\section{INTRODUÇÃO}

O processo de globalização tem promovido um aumento substancial na importância da produtividade, que eleva o nível de exigência tanto das pessoas como das organizações, transformando a qualidade em matéria aplicada ${ }^{1}$.

A qualidade dos processos em saúde vem sendo discutida entre os profissionais da área, cujos principais desafios estão na busca de uma assistência de excelência que visam atender às demandas de forma eficiente ${ }^{2}$. Nesse contexto, a qualidade, de modo geral, deve ser entendida como uma atitude coletiva, por se tratar de um diferencial técnico e social necessário, que envolve não somente o usuário do sistema, mas também os seus gestores ${ }^{3}$.

A melhoria da qualidade assistencial deve ser considerada pelos enfermeiros como um processo dinâmico e exaustivo de identificação dos fatores intervenientes no processo de trabalho, requerendo a implementação de ações e a elaboração de instrumentos que possibilitem avaliar, de maneira sistemática, os níveis de qualidade dos cuidados prestados. Desse modo, o enfermeiro precisa analisar os resultados da assistência para (re)definir as estratégias gerenciais ${ }^{4}$.

Entretanto, para que tais profissionais possam elaborar instrumentos que permitam avaliar os resultados de suas ações, é necessário um embasamento em informações que traduzam a realidade da assistência de forma direta ou indireta 5 .

No Brasil, a utilização de indicadores, embora seja de extrema importância para a gestão dos serviços, ainda possui uma lacuna no que diz respeito aos indicadores que representam a qualidade da assistência de Enfermagem em instituições hospitalares. Na década de 1990, os indicadores então utilizados em hospitais norte-americanos foram adotados no Brasil, porém eram pouco condizentes com a realidade daquele momento. Posteriormente, iniciativas foram surgindo, objetivando a adequação dos indicadores à realidade brasileira. Pode-se citar como importante publicação de referenciais para indicadores de Enfermagem os manuais do Programa "Compromisso com a Qualidade Hospitalar" (CQH) ${ }^{3}$.

O Centro Cirúrgico (CC) é considerado uma das unidades mais importantes, complexas e específicas do setor hospitalar, no qual são compartilhados inúmeros processos e subprocessos, ligados direta ou indiretamente à produção de cirurgias, por vezes estressantes, que podem afetar a qualidade da assistência prestada ${ }^{6}$.
Assim, observa-se uma crescente preocupação dos profissionais alocados no CC em compreender a complexidade que envolve a atuação do enfermeiro e a construção e validação dos indicadores de qualidade que norteiam suas ações ${ }^{3,7}$.

\section{OBJETIVOS}

Conhecer, caracterizar e analisar os principais temas nas literaturas nacional e internacional referentes aos indicadores de qualidade em Enfermagem, com ênfase no CC.

\section{MÉTODOS}

Trata-se de uma revisão integrativa, compreendida como parte valiosa do processo de criar e organizar a leitura com os mesmos níveis de clareza, rigor e replicação das pesquisas primárias ${ }^{8}$.

Tal método é composto por várias etapas recomendadas por especialistas na temática ${ }^{8,9}$. Para esta revisão, buscou-se: identificar o problema (definindo o tema da revisão em forma de questão-pergunta norteadora); selecionar a amostra (após o estabelecimento dos critérios de inclusão/ exclusão); definir as características da pesquisa (por meio da categorização dos estudos e da coleta de dados); avaliar/analisar os estudos incluídos na revisão, identificando similaridades e conflitos; discutir e interpretar os resultados e apresentar a revisão / síntese do conhecimento ${ }^{8,9}$.

A pergunta norteadora foi: qual foi o conhecimento produzido e publicado sobre o uso e a importância dos indicadores de qualidade em Enfermagem com ênfase no CC?

A busca dos artigos e a seleção da amostra ocorreram em maio de 2016, e o instrumento principal utilizado foi a busca em bancos de dados on-line da área da saúde: Literatura Latino-Americana e do Caribe em Ciências da Saúde (LILACS), Scientific Electronic Library Online (SciELO) e US National Library of Medicine (PubMed), com os descritores: indicadores de qualidade em assistência à saúde $A N D$ enfermagem $A N D$ gerenciamento da prática profissional, nas bases de dados brasileiras; e quality indicators AND nursing AND surgical center, nas internacionais.

Com a delimitação dos descritores, foi possível selecionar a amostra inicial da pesquisa, que adotou os seguintes critérios de inclusão: artigos na íntegra, encontrados nas literaturas nacional e internacional, publicados no período de janeiro de 2009 a maio de 2016. 
A amostra inicial constituiu-se de 87 artigos, sendo 35 encontrados na base de dados LILACS, 24 na SciELO e 28 na PubMed.

Após a seleção dos artigos, realizou-se leitura criteriosa do título e do resumo e, em seguida, foram excluídos aqueles que não respondiam à pergunta norteadora e que se repetiam entre as bases de dados.

Para a análise e posterior síntese dos artigos que atenderam aos critérios de inclusão, elaborou-se um instrumento para coleta de dados, baseado em literatura pertinente ${ }^{10} \mathrm{e}$ adaptado à realidade da pesquisa. Este contempla dados referentes ao pesquisador principal; à publicação (tipo de estudo, fonte, ano e país de origem); à instituição sede; à base de dados; ao objetivo; ao referencial metodológico e às principais conclusões.

\section{RESULTADOS}

Da leitura sistemática sobre o objeto abordado, obteve-se uma amostra final de 17 artigos científicos, sendo nove encontrados na LILACS, dois na SciELO e seis na PubMed. As publicações variaram entre 2009 e 2016, sendo que 2013 teve o maior número de contribuições (quatro artigos), seguido de 2011 e 2015 (com três publicações cada). Os enfermeiros foram os responsáveis pelo maior número de publicações (15 artigos), sendo 6 mestres, 4 doutores, 3 com pós-doutorado, 1 especialista e 3 sem informações da titulação, todos atuando em áreas distintas do CC.

O cenário principal dos estudos foram os hospitais, dentre eles nove públicos, um privado e seis sem especificação quanto a esse aspecto. Um dos artigos baseou-se nos dados fornecidos pelo Banco de Dados Nacional de Enfermagem de Indicadores de Qualidade (NDNQI).

A leitura possibilitou categorizar os principais assuntos abordados nos artigos, com destaque para a importância, a confiabilidade e os benefícios dos indicadores de qualidade para o gerenciamento da assistência de Enfermagem; os indicadores de qualidade de maior relevância para a assistência de Enfermagem em CC; as principais dificuldades relacionadas ao uso dos indicadores de qualidade; a importância da utilização dos sistemas informatizados para o uso dos indicadores de qualidade, evidenciando experiências nessa vertente por parte das organizações de saúde, e a visão dos enfermeiros sobre o uso dessa ferramenta de qualidade.

Embora os indicadores de qualidade específicos ao CC tenham sido pouco abordados nos artigos encontrados, é notório que esses são de grande importância para os processos de trabalho na referida unidade. Dentre os indicadores mais relevantes para a assistência de Enfermagem nesse cenário, a pesquisa revelou aqueles relacionados à: sistematização da assistência de Enfermagem perioperatória (SAEP), visita pré-operatória de Enfermagem (VPOE), lesão de pele (LP), queda, infecção em sítio cirúrgico (ISC) e aos registros de Enfermagem.

Vários fatores foram apontados como obstáculos para o uso dos indicadores de qualidade, dentre eles: o acúmulo de atividades privativas do enfermeiro na instituição; a falta de conhecimento teórico e prático do tema; o pouco conhecimento da gestão por processo; o pouco envolvimento da equipe na coleta de dados e a falta de acompanhamento dos gestores durante a implantação e utilização dos indicadores ${ }^{11}$.

Entre os estudos, evidenciou-se a preocupação em verificar a opinião dos enfermeiros sobre o emprego dos indicadores para avaliar a qualidade dos processos assistenciais das equipes de trabalho, uma vez que esses são os principais responsáveis pela elaboração dos instrumentos da coleta de dados, implantação e análise dos resultados.

O Quadro $1^{2,4,6-7,12,13-24}$ apresenta os dados quantitativos e qualitativos referentes aos artigos que compuseram a amostra.

Quadro 1. Dados quantitativos e qualitativos referentes aos artigos selecionados para a amostra da pesquisa.

\begin{tabular}{|c|c|c|c|}
\hline Título (País) & $\begin{array}{l}\text { Autores, Tipo de } \\
\text { estudo e Periódico }\end{array}$ & Objetivos & Conclusões \\
\hline $\begin{array}{l}\text { "Indicadores de qualidade } \\
\text { na assistência de terapia } \\
\text { intravenosa em um } \\
\text { hospital universitário: } \\
\text { uma contribuição da } \\
\text { Enfermagem" (Brasil)² }\end{array}$ & $\begin{array}{l}\text { Barbosa MT, Alves VH, } \\
\text { Rodrigues DP, Branco MB, } \\
\text { Souza RM, Bonazzi VC } \\
\text { Estudo quantitativo } \\
\text { Journal of Research } \\
\text { Fundamental Care Online }\end{array}$ & $\begin{array}{l}\text { Compreender os indicadores } \\
\text { de qualidade da assistência de } \\
\text { terapia intravenosa na Unidade } \\
\text { de Terapia Intensiva Neonatal } \\
\text { de um hospital universitário. }\end{array}$ & $\begin{array}{l}\text { O profissional de saúde deve facilitar e } \\
\text { promover a segurança do paciente em } \\
\text { prol de seu bem-estar e qualidade de vida, } \\
\text { evitando-se riscos e efeitos adversos. }\end{array}$ \\
\hline
\end{tabular}


Quadro 1. Continuação.

\begin{tabular}{|c|c|c|c|}
\hline Título (País) & $\begin{array}{l}\text { Autores, Tipo de } \\
\text { estudo e Periódico }\end{array}$ & Objetivos & Conclusões \\
\hline $\begin{array}{l}\text { "Opinião dos enfermeiros } \\
\text { sobre a utilização dos } \\
\text { indicadores de qualidade } \\
\text { na assistência de } \\
\text { Enfermagem" (Brasil) })^{12}\end{array}$ & $\begin{array}{l}\text { Silveira TV, Prado Júnior PP, } \\
\text { Siman AG, Amaro MO } \\
\text { Estudo qualitativo } \\
\text { Revista Gaúcha de } \\
\text { Enfermagem }\end{array}$ & $\begin{array}{l}\text { Investigar a opinião dos } \\
\text { enfermeiros de instituições } \\
\text { hospitalares sobre a } \\
\text { utilização dos indicadores de } \\
\text { qualidade da assistência de } \\
\text { Enfermagem. }\end{array}$ & $\begin{array}{l}\text { Dificuldades na utilização de indicadores: } \\
\text { falta de tempo, número inadequado de } \\
\text { profissionais e falta de conhecimento } \\
\text { sobre o tema. Os enfermeiros entendem } \\
\text { os indicadores como instrumentos } \\
\text { de avaliação e melhorias; entretanto, } \\
\text { possuem informação incompleta e } \\
\text { fragmentada sobre o seu uso. }\end{array}$ \\
\hline $\begin{array}{l}\text { "Improving patient safety } \\
\text { by optimizing the use of } \\
\text { Nursing human resources" } \\
\text { (Canadá) }{ }^{16}\end{array}$ & $\begin{array}{l}\text { Rochefort CM, Buckeridge } \\
\text { DL, Abrahamowicz M } \\
\text { Estudo de coorte } \\
\text { Implementation Science }\end{array}$ & $\begin{array}{l}\text { Determinar se os níveis do } \\
\text { pessoal de Enfermagem } \\
\text { estão associados com } \\
\text { um risco aumentado } \\
\text { de eventos adversos; } \\
\text { se o risco de eventos } \\
\text { adversos em relação } \\
\text { aos níveis do pessoal de } \\
\text { Enfermagem é modificado } \\
\text { pela complexidade das } \\
\text { necessidades dos pacientes; } \\
\text { e a possibilidade de se } \\
\text { estabelecerem os níveis } \\
\text { ideais para o pessoal de } \\
\text { Enfermagem. }\end{array}$ & $\begin{array}{l}\text { Estudo pioneiro na descrição do } \\
\text { efeito das estratégias da equipe de } \\
\text { Enfermagem sobre os riscos de eventos } \\
\text { adversos, como exposições variáveis no } \\
\text { tempo, permitindo determinar se esses } \\
\text { riscos são alterados conforme a duração } \\
\text { da exposição do pessoal (uso extensivo } \\
\text { de horas extras) ou a intensidade da } \\
\text { exposição (baixo volume de horas } \\
\text { de Enfermagem por paciente). Esses } \\
\text { dados auxiliaram na identificação dos } \\
\text { padrões de pessoal de Enfermagem, } \\
\text { gerando informações baseadas em } \\
\text { evidências capazes de auxiliarem os } \\
\text { gestores na tomada de decisões quanto } \\
\text { à utilização eficaz dos recursos escassos } \\
\text { de Enfermagem e na identificação dos } \\
\text { padrões de pessoal que minimizam o } \\
\text { risco de eventos adversos. }\end{array}$ \\
\hline $\begin{array}{l}\text { "Changes in patient and } \\
\text { nurse outcomes associated } \\
\text { with magnet hospital } \\
\text { recognition" (Estados Unidos } \\
\text { da América) }{ }^{17}\end{array}$ & $\begin{array}{l}\text { Kutney-Lee A, Stimpfel } \\
\text { AW, Sloane DM, Cimiotti JP, } \\
\text { Quinn LW, Aiken LH } \\
\text { Estudo quantitativo } \\
\text { Medical Care }\end{array}$ & $\begin{array}{l}\text { Comparar a qualidade dos } \\
\text { resultados apresentados } \\
\text { pelos pacientes cirúrgicos } \\
\text { em hospitais que obtiveram } \\
\text { o reconhecimento de } \\
\text { hospitais magnéticos/imãs, } \\
\text { entre } 1999 \text { e } 2007, \text { com } \\
\text { aqueles que se mantiveram } \\
\text { sem o título. }\end{array}$ & $\begin{array}{c}\text { Em geral, o reconhecimento } \\
\text { magnético/imã está associado a } \\
\text { melhorias significativas ao longo do } \\
\text { tempo relacionadas à qualidade do } \\
\text { ambiente de trabalho e voltadas ao } \\
\text { paciente e à Enfermagem, superando } \\
\text { os resultados obtidos pelos hospitais } \\
\text { não magnéticos/imãs. }\end{array}$ \\
\hline $\begin{array}{l}\text { "Fidedignidade de } \\
\text { indicadores de qualidade do } \\
\text { cuidado de Enfermagem: } \\
\text { testando a concordância } \\
\text { e confiabilidade inter } \\
\text { avaliadores" (Brasil) }\end{array}$ & $\begin{array}{c}\text { Vituri DW, Évora YD } \\
\text { Estudo quantitativo } \\
\text { Revista Latino-Americana } \\
\text { de Enfermagem }\end{array}$ & $\begin{array}{l}\text { Testar a concordância } \\
\text { e a confiabilidade de } 15 \\
\text { indicadores de qualidade da } \\
\text { assistência de Enfermagem } \\
\text { e validar tais instrumentos. }\end{array}$ & $\begin{array}{l}\text { Os indicadores apresentam excelente } \\
\text { confiabilidade e reprodutibilidade, } \\
\text { evidenciando que o desenvolvimento } \\
\text { de instrumentos de avaliação válidos } \\
\text { e fidedignos é possível, além de } \\
\text { imprescindíveis para o gerenciamento da } \\
\text { assistência de Enfermagem. }\end{array}$ \\
\hline $\begin{array}{l}\text { "Nurse staffing and } \\
\text { education and hospital } \\
\text { mortality in nine European } \\
\text { countries: a retrospective } \\
\text { observational study" } \\
\text { (Bélgica, Inglaterra, } \\
\text { Finlândia, Irlanda, Holanda, } \\
\text { Noruega, Espanha, Suécia } \\
\text { e Suíça) }{ }^{19}\end{array}$ & $\begin{array}{l}\text { Aiken LH, Sloane DM, } \\
\text { Bruyneel L, Heede KV, } \\
\text { Griffiths P, Busse R, et al. } \\
\text { Estudo quantitativo } \\
\text { The Lancet }\end{array}$ & $\begin{array}{l}\text { Avaliar as taxas de } \\
\text { mortalidade hospitalar após } \\
\text { procedimentos cirúrgicos } \\
\text { em relação à sobrecarga } \\
\text { de trabalho e ao nível de } \\
\text { formação do profissional de } \\
\text { Enfermagem. }\end{array}$ & $\begin{array}{l}\text { A sobrecarga de trabalho do profissional } \\
\text { de Enfermagem aumentou a probabilidade } \\
\text { de óbito de pacientes internados para } \\
7 \% \text {, dentro de } 30 \text { dias de internação. } \\
0 \text { aumento do nível educacional dos } \\
\text { enfermeiros associou-se à diminuição } \\
\text { dessa taxa. Assim, reduções na equipe } \\
\text { de Enfermagem para economia podem } \\
\text { influenciar os resultados dos cuidados de } \\
\text { pacientes. Nível educacional superior pode } \\
\text { reduzir mortes hospitalares estáveis. }\end{array}$ \\
\hline
\end{tabular}


Quadro 1. Continuação.

\begin{tabular}{|c|c|c|c|}
\hline Título (País) & $\begin{array}{l}\text { Autores, Tipo de } \\
\text { estudo e Periódico }\end{array}$ & Objetivos & Conclusões \\
\hline $\begin{array}{l}\text { “Indicadores de qualidade da } \\
\text { assistência de Enfermagem } \\
\text { em centro cirúrgico: revisão } \\
\text { integrativa de literatura” } \\
\text { (Brasil) }\end{array}$ & $\begin{array}{l}\text { Santos MC, Rennó CS } \\
\text { Revisão integrativa da } \\
\text { literatura } \\
\text { Revista de Administração } \\
\text { em Saúde }\end{array}$ & $\begin{array}{l}\text { Identificar os indicadores de } \\
\text { qualidade da assistência em } \\
\text { Centro Cirúrgico. }\end{array}$ & $\begin{array}{l}\text { Os indicadores mais relevantes } \\
\text { encontrados com a revisão } \\
\text { relacionaram-se à SAEP, à VPOE, LP, } \\
\text { queda, ISC e registro completo. }\end{array}$ \\
\hline $\begin{array}{l}\text { "Dificuldades vivenciadas } \\
\text { pelo enfermeiro na } \\
\text { utilização de indicadores de } \\
\text { processo" (Brasil) }\end{array}$ & $\begin{array}{c}\text { Menezes PI, D' Innocenzo M } \\
\text { Estudo quantitativo } \\
\text { Revista Brasileira de } \\
\text { Enfermagem }\end{array}$ & $\begin{array}{l}\text { Identificar as dificuldades } \\
\text { vivenciadas pelos } \\
\text { enfermeiros da Santa Casa de } \\
\text { Montes Claros na utilização } \\
\text { dos indicadores de processos } \\
\text { assistenciais (em qualquer } \\
\text { fase ou etapa do processo). }\end{array}$ & $\begin{array}{l}\text { Entre as dificuldades encontradas, } \\
\text { destacaram-se acúmulo de atividades } \\
\text { privativas ( } 61,5 \%) \text {; falta de conhecimento } \\
\text { teórico e prático sobre o tema ( } 46,2 \%) \text {; } \\
\text { pouco conhecimento sobre a gestão por } \\
\text { processo ( } 43,6 \%) \text {; pouco envolvimento da } \\
\text { equipe na coleta de dados ( } 30,8 \%) \text {, dentre } \\
\text { outros. } 0 \text { estudo concluiu ser importante } \\
\text { refletir sobre as atividades privativas do } \\
\text { enfermeiro dentro das unidades de saúde } \\
\text { e a reorganização do seu processo de } \\
\text { trabalho, além da inserção do tema nos } \\
\text { cursos de graduação em Enfermagem. }\end{array}$ \\
\hline $\begin{array}{l}\text { "Falls among adult patients } \\
\text { hospitalized in the United } \\
\text { States: prevalence and trends" } \\
\text { (Estados Unidos da América) }{ }^{20}\end{array}$ & $\begin{array}{l}\text { Bouldin ED, Andresen } \\
\text { EM, Dunton NE, Simon M, } \\
\text { Waters TM, Liu M, et al. } \\
\text { Estudo quantitativo } \\
\text { Journal of Patient Safety }\end{array}$ & $\begin{array}{l}\text { Fornecer dados sobre a } \\
\text { prevalência de quedas em } \\
\text { unidades médico, cirúrgico } \\
\text { e médico-cirúrgico em } \\
\text { hospitais de cuidados } \\
\text { agudos dos EUA, bem como } \\
\text { determinar a ocorrência } \\
\text { de quedas durante os } 27 \\
\text { meses que antecederam } \\
\text { a implementação da nova } \\
\text { limitação de reembolso } \\
\text { dos Centros de Serviços } \\
\text { Médicos, de acordo com as } \\
\text { condições adquiridas } \\
\text { no hospital. }\end{array}$ & $\begin{array}{l}\text { O trabalho fornece a primeira avaliação } \\
\text { nacional de quedas, incluindo aquelas } \\
\text { que resultaram em prejuízo para } \\
\text { as referidas unidades. As unidades } \\
\text { de clínica médica apresentaram } \\
\text { maior número de quedas devido aos } \\
\text { diagnósticos mais complexos e ao fato } \\
\text { de os pacientes deambularem. }\end{array}$ \\
\hline $\begin{array}{l}\text { "Associations between rates } \\
\text { of unassisted inpatient falls } \\
\text { and levels of registered and } \\
\text { non-registered nurse staffing" } \\
\text { (Estados Unidos da América) }{ }^{21}\end{array}$ & $\begin{array}{l}\text { Staggs VS, Dunton N } \\
\text { Estudo quantitativo } \\
\text { International Journal for } \\
\text { Quality in Health Care }\end{array}$ & $\begin{array}{l}\text { Melhorar a compreensão } \\
\text { da equipe de Enfermagem } \\
\text { sobre as quedas não } \\
\text { assistidas, explorando as } \\
\text { associações não lineares } \\
\text { entre as taxas de queda não } \\
\text { assistidas e as categorias } \\
\text { de Enfermagem, permitindo } \\
\text { aos gestores a melhora da } \\
\text { segurança do paciente. }\end{array}$ & $\begin{array}{l}\text { O aumento de profissionais de nível } \\
\text { técnico se mostra ineficaz na prevenção } \\
\text { de quedas desassistidas. } 0 \text { aumento } \\
\text { de enfermeiros pode ser eficaz, } \\
\text { dependendo do tipo de unidade. }\end{array}$ \\
\hline $\begin{array}{l}\text { "Nurse reported quality of care: } \\
\text { a measure of hospital quality" } \\
\text { (Estados Unidos da América) }{ }^{22}\end{array}$ & $\begin{array}{c}\text { McHugh MD, Stimpfel AW } \\
\text { Estudo quantitativo } \\
\text { Research in Nursing \& } \\
\text { Health }\end{array}$ & $\begin{array}{l}\text { Avaliar a visão dos } \\
\text { enfermeiros no que se } \\
\text { refere à qualidade do } \\
\text { atendimento. }\end{array}$ & $\begin{array}{l}\text { 10,0\% dos enfermeiros relataram que } \\
\text { a maior qualidade do atendimento } \\
\text { foi associada com a menor chance } \\
\text { de mortalidade e incapacidade } \\
\text { de recuperação; maior satisfação } \\
\text { do paciente e maior processo de } \\
\text { acolhimento no infarto agudo do } \\
\text { miocárdio, pneumonia e a pacientes } \\
\text { cirúrgicos, sendo assim a qualidade dos } \\
\text { cuidados um importante indicador de } \\
\text { desempenho hospitalar. }\end{array}$ \\
\hline
\end{tabular}


Quadro 1. Continuação.

\begin{tabular}{|c|c|c|c|}
\hline Título (País) & $\begin{array}{l}\text { Autores, Tipo de } \\
\text { estudo e Periódico }\end{array}$ & Objetivos & Conclusões \\
\hline $\begin{array}{l}\text { "Mensuração de indicadores de } \\
\text { qualidade em centro cirúrgico: } \\
\text { tempo de limpeza e intervalo } \\
\text { entre cirurgias" (Brasil) }\end{array}$ & $\begin{array}{c}\text { Jericó MC, Perroca MG, } \\
\text { Penha VC } \\
\text { Estudo quantitativo } \\
\text { Revista Latino-Americana } \\
\text { de Enfermagem }\end{array}$ & $\begin{array}{c}\text { Mensurar o tempo } \\
\text { despendido para limpeza } \\
\text { concorrente realizada pelo } \\
\text { serviço de higiene e limpeza } \\
\text { em sala cirúrgica, e o } \\
\text { intervalo entre as cirurgias, } \\
\text { bem como investigar a } \\
\text { associação entre tempo } \\
\text { de limpeza, porte e } \\
\text { especialidade da cirurgia, } \\
\text { período de ocorrência e } \\
\text { tamanho da sala. }\end{array}$ & $\begin{array}{l}0 \text { tempo de limpeza da sala cirúrgica } \\
\text { foi de } 7,1 \text { minutos e o do intervalo entre } \\
\text { as cirurgias, } 35,6 \text { minutos. Não foi } \\
\text { encontrada correlação entre o tempo } \\
\text { de limpeza e as demais variáveis. } \\
\text { Esses achados instrumentalizam } \\
\text { os enfermeiros no uso eficiente de } \\
\text { recursos, agilizando o processo de } \\
\text { trabalho em Centro Cirúrgico. }\end{array}$ \\
\hline $\begin{array}{l}\text { "Sistema informatizado } \\
\text { para gerenciamento de } \\
\text { indicadores da assistência } \\
\text { de Enfermagem do Hospital } \\
\text { São Paulo" (Brasil) }{ }^{14}\end{array}$ & $\begin{array}{c}\text { Labbadia LL, D' Innocenzo } \\
\text { M, Fogliano RR, Silva GE, } \\
\text { Queiroz RM, Carmagnani } \\
\text { MI, et al. } \\
\text { Relato de caso } \\
\text { Revista Escola de } \\
\text { Enfermagem da USP }\end{array}$ & $\begin{array}{l}\text { Descrever a experiência de } \\
\text { um grupo de enfermeiros } \\
\text { na criação de um } \\
\text { sistema informatizado } \\
\text { desenvolvido no } \\
\text { Hospital São Paulo para } \\
\text { o gerenciamento dos } \\
\text { indicadores da assistência } \\
\text { de Enfermagem. }\end{array}$ & $\begin{array}{l}\text { O sistema informatizado permite } \\
\text { armazenar dados pertinentes } \\
\text { aos processos assistenciais de } \\
\text { Enfermagem, sendo disponibilizado } \\
\text { para a avaliação dos resultados da } \\
\text { assistência de Enfermagem, os quais } \\
\text { podem ser consultados e impressos no } \\
\text { momento necessário. }\end{array}$ \\
\hline $\begin{array}{l}\text { "Utilização de indicadores } \\
\text { de desempenho em serviço } \\
\text { de enfermagem de hospital } \\
\text { público" (Brasil) }\end{array}$ & $\begin{array}{l}\text { Gabriel CS, Melo MR, Rocha } \\
\text { FL, Bernardes A, Miguelaci } \\
\text { T, Silva ML } \\
\text { Estudo quantitativo } \\
\text { Revista Latino-Americana } \\
\text { de Enfermagem }\end{array}$ & $\begin{array}{l}\text { Identificar os indicadores de } \\
\text { desempenho, adotados pelo } \\
\text { serviço de Enfermagem } \\
\text { de um hospital público, } \\
\text { e analisar a opinião dos } \\
\text { enfermeiros em relação } \\
\text { à utilização desses } \\
\text { indicadores para avaliar a } \\
\text { qualidade da assistência. }\end{array}$ & $\begin{array}{l}\text { Tal instituição utiliza os indicadores para } \\
\text { o acompanhamento de resultados, e há } \\
\text { valorização da utilização de indicadores } \\
\text { de processos pelos enfermeiros para } \\
\text { avaliar o desempenho da Enfermagem, } \\
\text { sendo necessário ampliar a análise para } \\
\text { indicadores multidisciplinares. }\end{array}$ \\
\hline $\begin{array}{l}\text { "Percepção dos enfermeiros } \\
\text { sobre os resultados dos } \\
\text { indicadores de qualidade } \\
\text { na melhoria da prática } \\
\text { assistencial" (Brasil) }{ }^{15}\end{array}$ & $\begin{array}{l}\text { Franco JN, Barros BP, } \\
\text { Vaidotas M, D'Innocenzo M } \\
\text { Estudo quali-quantitativo } \\
\text { Revista Brasileira de } \\
\text { Enfermagem }\end{array}$ & $\begin{array}{l}\text { Diagnosticar a percepção } \\
\text { dos enfermeiros de um } \\
\text { hospital privado sobre os } \\
\text { processos de qualidade em } \\
\text { Enfermagem aplicados à } \\
\text { melhoria da assistência. }\end{array}$ & $\begin{array}{l}\text { Os enfermeiros consideraram a } \\
\text { utilização dos resultados de indicadores } \\
\text { um instrumento estratégico, e sua } \\
\text { percepção corrobora a prática da } \\
\text { melhoria assistencial por meio da } \\
\text { utilização de ferramentas e indicadores } \\
\text { com ênfase no registro de falhas no } \\
\text { processo como possíveis sugestões de } \\
\text { melhoria da qualidade. }\end{array}$ \\
\hline $\begin{array}{l}\text { "Validação de conteúdo de } \\
\text { indicadores de qualidade } \\
\text { para avaliação do cuidado de } \\
\text { Enfermagem" (Brasil) }\end{array}$ & $\begin{array}{l}\text { Vituri DW, Matsuda LM } \\
\text { Estudo quantitativo } \\
\text { Revista Escola de } \\
\text { Enfermagem da USP }\end{array}$ & $\begin{array}{l}\text { Validar o conteúdo de um } \\
\text { instrumento de avaliação } \\
\text { do cuidado de Enfermagem } \\
\text { composto por indicadores } \\
\text { de qualidade do cuidado de } \\
\text { Enfermagem na prevenção } \\
\text { de efeitos adversos - } \\
\text { validação de instrumento. }\end{array}$ & $\begin{array}{l}\text { A partir dos resultados, o estudo } \\
\text { acredita no procedimento de validação } \\
\text { do conteúdo como imprescindível para o } \\
\text { desenvolvimento de medidas avaliativas. }\end{array}$ \\
\hline $\begin{array}{l}\text { "La utilización de } \\
\text { herramientas de } \\
\text { investigación cualitativa en } \\
\text { la construcción y diseño de } \\
\text { indicadores de la práctica de } \\
\text { enfermería" (Chile) }{ }^{24}\end{array}$ & $\begin{array}{l}\text { Jara PC, Valenzuela SS } \\
\text { Estudo qualitativo } \\
\text { Ciencia y Enfermería }\end{array}$ & $\begin{array}{l}\text { Descrever o processo dos } \\
\text { indicadores na prática de } \\
\text { Enfermagem em Unidade de } \\
\text { Terapia Intensiva, utilizando } \\
\text { abordagens qualitativas. }\end{array}$ & $\begin{array}{l}\text { Possibilitou a descrição mais próxima da } \\
\text { prática de Enfermagem e identificação } \\
\text { de indicadores específicos. }\end{array}$ \\
\hline
\end{tabular}

SAEP: sistematização da assistência de Enfermagem perioperatória; VPOE: visita pré-operatória de Enfermagem; LP: lesão de pele; ISC: infecção em sítio cirúrgico. 


\section{DISCUSSÃO}

Esta pesquisa demonstra que o enfermeiro se destaca não apenas em quantidade de publicações, mas também nas atividades pertinentes ao gerenciamento dos processos de trabalho. É o profissional habilitado para gerir as necessidades que envolvem o ato anestésico-cirúrgico em todas as suas etapas, sendo-lhe atribuídas atividades relativas ao funcionamento da unidade, bem como atividades técnicas, administrativas, assistenciais e de gestão de pessoas ${ }^{25}$.

Dessa forma, torna-se importante a participação do enfermeiro gestor no processo de avaliação da qualidade e produtividade no CC, com o propósito de detectar e redesenhar atividades que não estão em conformidade com padrões e requisitos que visam à proteção da saúde individual e coletiva, realizar seu monitoramento contínuo e comparar seus resultados com aqueles encontrados nos hospitais de melhores práticas ${ }^{6}$.

O setor hospitalar, cenário principal dos estudos, caracteriza-se como um dos mais complexos e de difícil gerenciamento, comportando alto risco inerente à atividade, o que impõe aos gestores medições constantes e variadas, levando-os à implantação de indicadores de qualidade em diversas áreas de abrangência ${ }^{11}$.

A pesquisa quantitativa utilizada em grande parte dos artigos que compõem a amostra "compreende a coleta sistemática de informações numéricas, preservando as condições de controle, além da análise dessa informação por meio de procedimentos estatísticos" ${ }^{326}$.

Dentre os principais assuntos abordados nos estudos analisados, o reconhecimento da importância, da confiabilidade e dos benefícios trazidos pelo uso dos indicadores de qualidade para o gerenciamento da assistência de Enfermagem aparece em destaque, visto que a utilização desses dados permite o estabelecimento de padrões, bem como o acompanhamento de sua evolução ao longo dos anos. Embora o uso de um único indicador isoladamente não possibilite o conhecimento da complexidade da realidade social, a associação de vários deles e, ainda, a comparação entre diferentes indicadores de distintas localidades facilitam sua compreensão ${ }^{27}$. Os indicadores de qualidade baseiam-se na conformidade dos padrões estabelecidos para monitorar os processos e resultados ${ }^{28}$.

Diante dessa capacidade de abrangência dos indicadores de qualidade, são notórias a preocupação, a necessidade e o empenho dos profissionais de Enfermagem em construir e validar instrumentos que os contenham e que permitam balizar suas ações para a tomada de decisões, na busca constante da qualidade dos seus processos de trabalho, refletindo os diferentes contextos de sua prática profissional.

Embora as publicações referentes aos indicadores de qualidade em CC sejam escassas, nota-se a importância dada ao processo de sistematização, que é representado pelo SAEP, uma vez que possibilita a quantificação da assistência prestada pela equipe de Enfermagem ${ }^{27}$.

O SAEP abrange a VPOE, que permite ao enfermeiro do CC conhecer o seu paciente com antecedência, traçar planos de cuidados e fornecer as informações necessárias, diminuindo, com isso, o estresse e a ansiedade com relação ao procedimento a ser realizado ${ }^{29}$. Esse monitoramento da evolução do paciente e a detecção precoce de possíveis falhas na assistência de Enfermagem possibilitam cuidados ao paciente de forma holística ${ }^{7}$, pois o SAEP é uma forma sistematizada de cuidar, sem interrupções, respeitando o indivíduo quanto às suas experiências, problemas e expectativas ${ }^{29}$.

Outro indicador apontado como relevante e pertinente para avaliar a qualidade da assistência de Enfermagem foi a incidência de $\mathrm{LP}^{30}$. A prevenção desse evento sofre participação fundamental do enfermeiro que, frente à condição de dependência física e fragilidade do paciente, deve atuar de forma assertiva e atentar para o risco presente das LP a que esses pacientes estão expostos ${ }^{7}$.

O uso da eletrocirurgia também oferece riscos aos pacientes nesse ambiente, já que ocasiona queimaduras, explosões de misturas combustíveis, incluindo gases anestésicos e intestinais, estimulação de tecidos excitáveis e interferência sobre instrumentos e marca-passos. A queimadura, entretanto, é a complicação mais frequente ${ }^{31}$. Os riscos químicos também estão presentes no período perioperatório, representando perigo tanto para o paciente como para os profissionais que atuam nesse setor ${ }^{32}$.

O posicionamento do paciente frente a uma intervenção cirúrgica também exerce influência significativa na qualidade da assistência, tendo como principal finalidade promover acesso ao sítio cirúrgico, devendo ser realizado de forma correta, garantindo a segurança do paciente e prevenindo complicações ${ }^{33}$.

$\mathrm{O}$ indicador da incidência de queda do paciente no CC é considerado um evento grave, que pode ser explicado pela vigilância inadequada de pacientes sem condições físicas para deambular desacompanhados ou pela não aderência às orientações prestadas pela equipe de Enfermagem ${ }^{34}$. 
A ISC é considerada um indicador que, quando apresenta resultados elevados em cirurgias limpas, sinaliza falha técnica nos processos cirúrgicos, comprometendo a recuperação dos pacientes. É importante lembrar que as medidas de prevenção são de responsabilidade dos envolvidos na assistência ao paciente cirúrgico ${ }^{7}$.

Diante dos diversos riscos presentes no CC, cabe ao enfermeiro e à equipe cirúrgica vigilância constante, atuação de forma preventiva e planejamento de ações que garantam a segurança do paciente em todas as etapas do procedimento anestésico-cirúrgico. O "Segundo desafio global para a segurança do paciente: cirurgias seguras salvam vidas" é um protocolo que promove ações voltadas para a prevenção de eventos adversos, a fim de assegurar assistência livre de danos ${ }^{7}$.

Para o enfermeiro, um dos meios de avaliar a qualidade da assistência de Enfermagem no CC é o registro adequado de todas as atividades realizadas pela equipe, o que torna necessária a implantação de um indicador que avalie a eficácia desses registros ${ }^{7}$. Nesse sentido, os registros de Enfermagem devem ser considerados pela equipe de saúde um meio de comunicação escrita e efetiva, que facilita a coordenação e a continuidade do planejamento das atividades em saúde. Por esse motivo, devem ser escritos de forma coerente e completa.

Um dos estudos abordados na pesquisa, referente às dificuldades relacionadas ao uso dos indicadores de qualidade, corrobora as dificuldades descritas na literatura no que tange aos elementos constitutivos, tais como: falta de tempo, número inadequado de profissionais e falta de conhecimento a respeito do tema. Esse mesmo estudo demonstrou que, em relação ao emprego dos indicadores da assistência de Enfermagem, apenas $54,0 \%$ dos enfermeiros utilizaram esse método para orientar suas ações, ou seja, 46,0\% deles coletam e analisam os indicadores, mas não fazem uso da avaliação dos resultados para implementar melhorias nos setores em que trabalham, isto é, utilizam a métrica, mas não fazem uma abordagem qualitativa dos fenômenos investigados ${ }^{12}$.

Outro estudo, que igualmente corrobora os dados apresentados, notabiliza que vários profissionais mencionam não haver encontros para a apresentação dos resultados das avaliações por indicadores, bem como não ocorrer discussões dessas avaliações ${ }^{30}$.

A discussão sobre os indicadores tem assumido um papel de destaque na gestão dos serviços de Enfermagem ${ }^{35}$. Com isso, espera-se que esse momento seja adotado pela equipe de Enfermagem como uma estratégia para identificar fragilidades e estabelecer metas com vistas à melhoria da assistência prestada. Esse deve ser um trabalho conjunto entre gestores e trabalhadores envolvidos no cuidado ${ }^{30}$.

Outro dado revelado pela pesquisa é que os enfermeiros não possuem contato teórico e/ ou prático com o tema durante a graduação, não sendo o conteúdo contemplado nos currículos da maior parte dos estabelecimentos de ensino. Desse modo, a falta de informação é uma das razões da dificuldade dos enfermeiros utilizarem indicadores. Por conseguinte, há a necessidade urgente de que esse conteúdo seja revisto e considerado pelos cursos de graduação ${ }^{13}$.

Outro motivo pelo qual os enfermeiros relatam dificuldades em utilizar os indicadores de qualidade é o fato de desconhecerem a sua aplicação. Nesse sentido, é importante que os indicadores sejam compreensíveis pelos usuários da informação. Eles precisam ser gerados, manejados de forma regular e sistêmica, tornando-se instrumentos valiosos para a avaliação e gestão ${ }^{13}$.

Com relação ao acúmulo das atividades atribuídas ao enfermeiro dentro do hospital e, consequentemente, à sobrecarga de trabalho, ambas apontadas como principais causas dificultadoras de se trabalhar com indicadores, faz-se necessária uma reflexão que analise criticamente essas atividades, com o propósito de reorganizar o processo de trabalho desse profissional dentro de suas unidades ${ }^{13}$.

Em virtude das evidências, nota-se que o número reduzido dos profissionais de Enfermagem impede a implementação de ações com qualidade e segurança. Urge que a administração das instituições seja sensível a essa demanda, promovendo mudanças positivas para a instituição, o profissional e o paciente ${ }^{12}$.

Uma das formas de padronizar e facilitar a implantação, coleta e análise dos indicadores é através do uso de sistemas informatizados. Esses contam com bancos de dados interativos, interface intuitiva, de fácil manuseio e atrativa do ponto de vista pedagógico, tendo o enfermeiro uma moderna ferramenta de trabalho, capaz de mensurar e contribuir para a qualidade de seu serviço ${ }^{14}$.

Os enfermeiros, mesmo diante de tantas dificuldades relacionadas ao uso dos indicadores, consideram esses meios importantes para o desenvolvimento do trabalho e ponderam que o uso de seus resultados como instrumentos estratégicos reverte em melhorias para a prática assistencial, possibilitando a avaliação do desempenho da Enfermagem ${ }^{4,13,15}$.

Compete ao enfermeiro do CC implantar, analisar criticamente e monitorar os indicadores necessários para qualificar a assistência de Enfermagem, bem como promover a 
interação entre os profissionais envolvidos no procedimento anestésico-cirúrgico, de modo a prevenir os riscos e controlar as complicações ${ }^{29}$.

O envolvimento de todos os membros de uma instituição no processo de implantação e utilização dos indicadores de qualidade é a força que viabiliza o alcance de resultados satisfatórios dos processos de trabalho. Mais importante, garante a prestação de uma assistência de Enfermagem segura, com qualidade e baseada em evidências.

Considera-se, portanto, que o avanço deste estudo está em demonstrar o conhecimento produzido nessa temática, diminuindo a lacuna existente na literatura.

No entanto, a limitação desta pesquisa refere-se ao fato de a análise de literatura ser circunscrita a artigos publicados e disponíveis na íntegra, excluindo-se, assim, outras fontes de dados existentes.

\section{CONCLUSÃO}

A avaliação de desempenho das atividades do CC por meio de indicadores abre caminho para a revisão crítica dos principais processos, possibilitando a intervenção nos pontos falhos e o desenvolvimento de melhorias voltadas à assistência prestada aos pacientes.

Diante da lacuna na literatura sobre os indicadores de qualidade com ênfase em CC, observada com o desenvolvimento desta revisão integrativa, foi necessário o uso de artigos que traziam informações sobre indicadores gerais. Essa conjuntura denota a importância de ampliar a cultura da qualidade nos serviços de Enfermagem na referida área, capacitando enfermeiros para o desenvolvimento e a análise de indicadores e possibilitando uma reflexão sobre a assistência de forma dinâmica, com o objetivo de atingir a excelência do cuidado.

O empenho em desenvolver novos estudos que retratem o tema fornecerá subsídios e embasamento prático aos profissionais de Enfermagem, principalmente na mensuração da qualidade de seus processos, pontuando acertos e falhas que interferem diretamente nos seus cuidados, sejam eles diretos ou indiretos (de gestão), revertendo, assim, em melhoria da qualidade da assistência prestada no CC.

Vale ressaltar que esta revisão integrativa sobre os indicadores de qualidade em Enfermagem, com ênfase no CC, suscitou a elaboração de uma pesquisa pela autora principal no curso de Mestrado Profissional.

\section{REFERÊNCIAS}

1. Fusco SFB, Spiri WC. Análise dos indicadores de qualidade de centros de material e esterilização de hospitais públicos acreditados. Texto Contexto Enferm. 2014;23(2):426-33.

2. Barbosa MTSR, Alves VH, Rodrigues DP, Branco MBRL, Souza RMP, Bonazzi VCAM. Indicadores de qualidade na assistência de terapia intravenosa em um hospital universitário: uma contribuição da enfermagem. J Res Fundam Care Online. 2015;7(2):2277-86.

3. Programa de Qualidade Hospitalar (CQH). Manual de indicadores de enfermagem NAGEH. 2a ed. São Paulo: APM/CREMESP; 2012. 60 p.

4. Gabriel CS, Melo MRAC, Rocha FLR, Bernardes A, Miguelaci T, Silva MLP. Utilização de indicadores de desempenho em serviço de enfermagem de hospital público. Rev LatinoAm Enferm [Internet]. 2011;19(5):[09 telas]. [citado 2016 maio 25]. Disponível em: http:// www.scielo.br/pdf/rlae/v19n5/pt_24.pdf.

5. Kurcgant $P$, Tronchin DMR, Melleiro MM. A construção de indicadores de qualidade para a avaliação de recursos humanos nos serviços de enfermagem: pressupostos teóricos. Acta Paul Enferm. 2006;19(1):88-91.
6. Jericó MC, Perroca MG, Penha VC. Mensuração dos indicadores de qualidade em centro cirúrgico: tempo de limpeza e intervalo entre cirurgias. Rev LatinoAm Enferm. 2011;19(5):1239-46. [citado 2016 junho 30]. Disponível em: http://www.scielo.br/pdf/rlae/ v19n5/pt_23.pdf.

7. Santos MC, Rennó CSN. Indicadores de qualidade da assistência de enfermagem em centro cirúrgico: revisão integrativa da literatura. Rev Adm Saúde. 2013;15(58):27-36.

8. Ganong LH. Integrative reviews of nursing research. Res Nurs Health. 1987;10(1):1-11.

9. Whittemore R, Knafl K. The integrative review: update methodology. J Adv Nurs. 2005;52(5):546-53.

10. Ursi ES. Prevenção de lesões de pele no perioperatório: revisão integrativa da literatura. Rev LatinoAm Enferm. 2006;14(1):124-31.

11. Bittar OJNV. Indicadores de qualidade e quantidade em saúde. Rev Adm Saúde. 2004;6(22):15-8. 
12. Silveira TVL, Prado Júnior PP, Siman AG, Amaro MOF. Opinião dos enfermeiros sobre a utilização dos indicadores de qualidade na assistência de enfermagem. Rev Gaúcha Enferm. 2015;36(2):82-8.

13. Menezes PIFB, D'Innocenzo M. Dificuldades vivenciadas pelo enfermeiro na utilização de indicadores de processos. Rev Bras Enferm. 2013;66(4):571-7.

14. Labbadia LL, D'Innocenzo M, Fogliano RRF, Silva GEF, Queiroz RMRM, Carmagnani MIS, et al. Sistema informatizado para gerenciamento de indicadores da assistência de enfermagem do Hospital São Paulo. Rev Esc Enferm USP. 2011;45(4):1013-7.

15. Franco JN, Barros BPA, Vaidotas M, D'Innocenzo M. Percepção dos enfermeiros sobre os resultados dos indicadores de qualidade na melhoria da prática assistencial. Rev Bras Enferm. 2010;63(5):806-10.

16. Rochefort CM, Buckeridge DL, Wicz MA. Improving patient safety by optimizing the use of nursing human resources. Implementation Science. 2015;10(89):1-11. [citado 2016 maio 24]. Disponivel em: https://implementationscience.biomedcentral.com/ articles/10.1186/s13012-015-0278-1. DOI: 10.1186/s13012-015-0278-1.

17. Kutney-Lee A, Stimpfel AW, Sloane DM, Cimiotti JP, Quinn LW, Aiken LH. Changes in patient and nurse outcomes associated with magnet hospital recognition. Med Care. 2015; 53(6):550-7. [citado 2016 junho 01]. Disponivel em: https://www.ncbi.nlm.nih.gov/pubmed/25906016. DOI: 10.1097/MLR.0000000000000355.

18. Vituri DW, Évora YDM. Fidedignidade de indicadores de qualidade do cuidado de enfermagem: testando a concordância e confiabilidade Inter avaliadores. Rev. Latino-Am. Enfermagem. 2014;22(2):23440. [citado 2016 maio 27]. Disponível em: http://www.scielo. $\mathrm{br} / \mathrm{pdf} / \mathrm{rlae} / \mathrm{v} 22 \mathrm{n} 2 / \mathrm{pt} \_0104-1169-$ rlae-22-02-00234.pdf. DOI: 10.1590/0104-1169.3262.2407.

19. Aiken LH, Sloane DM, Bruyneel L, Heede KVD, Griffiths P, Busse R, Diomidous M, Kinnunen J, Kózka M, Lesaffre E, Casbas MMMTM, Rafferty AM, Schwendiman R, P Anne Scott, Tishelman C, Achterberg TV, Sermeus W, for the RN4CAST consortium. Nurse staffing and education and hospital mortality in nine European countries: a retrospective observational study. The Lancet. 2014;383(9931):18241830. [citado 2016 maio 22]. Disponível em: http://www.thelancet. com/pdfs/journals/lancet/PIIS0140-6736(13)62631-8.pdf. DOI: http:// dx.doi.org/10.1016/S0140-6736(13)62631-8.

20. Bouldin EL, Andresen EM, Dunton NE, Simon M, Waters TM, Liu M, Daniels MJ, Mion LC, Shorr RI. Falls among Adult Patients Hospitalized in the United States: prevalence and Trends. J Patient Saf. 2013;9(1):137. [citado 2016 maio 22]. Disponível em: https://www.ncbi.nlm.nih. gov/pubmed/23143749. DOI: 10.1097/PTS.0b013e3182699b64.

21. Staggs VS, Dunton N. Associations between rates of unassisted inpatient falls and levels of registered and non-registered nurse staffing. International Journal for Quality in Health Care. 2013;p1-6. [citado 2016 maio 15]. Disponível em: http://intqhc.oxfordjournals. org/content/intqhc/early/2013/11/12/intqhc.mzt080.full.pdf.
22. McHugh MD, Stimpfel AW. Nurse Reported Quality of Care: a Measure of Hospital Quality. Res Nurs Health. 2012;35(6):56675. [citado 2016 maio 16]. Disponível em: https://www.ncbi.nlm. nih.gov/pubmed/22911102. DOI: 10.1002/nur.21503. Epub 2012 Aug 21.

23. Vituri DW; Matsuda LM. Validação de conteúdo de indicadores de qualidade para avaliação do cuidado de enfermagem. Rev Esc Enferm USP. 2009;43(2):429-37. [citado 2016 junho 13]. Disponível em: http://www.scielo.br/pdf/reeusp/v43n2/a24v43n2.pdf.

24. Patrícia Jara C, Sandra Valenzuela S. La utilización de herramientas de investigación cualitativa en la construcción y diseño de indicadores de la práctica de enfermería. Ciencia y Enfermeria. 2009;15(3):9-13. [citado 2016 junho 10]. Disponível em: http://www.scielo.cl/pdf/cienf/ v15n3/art_02.pdf.

25. Sociedade Brasileira de Enfermagem em Centro Cirúrgico. Centro de Material e Esterilização, Centro Cirúrgico e Recuperação pós-anestésica. Práticas recomendadas. 6a ed. São Paulo: SOBECC; 2013.

26. Polit DF, Beck CT. Fundamentos de pesquisa em enfermagem. Porto Alegre: Artmed; 2011.

27. Possari JF. Centro cirúrgico: planejamento, organização e gestão. 5a ed. São Paulo: látria; 2011.

28. Luongo J, organizadora. Gestão de qualidade em saúde. São Paulo: Rideel; 2011.

29. Bispo LGLL, Maria VLR. Indicadores de qualidade da assistência de enfermagem em centro cirúrgico. Rev SOBECC. 2010;15(1):30-6.

30. Cavalcante PS, Rossaneis MA, Haddad MCL, Gabriel CS. Indicadores de qualidade utilizados no gerenciamento da assistência de enfermagem hospitalar. Rev Enferm UERJ. 2015;23(6):787-93.

31. Afonso CT, Silva AL, Fabrini DS, Afonso CT, Côrtes MGW, Sant'anna LL. Risco do uso do eletrocautério em pacientes portadores de adornos metálicos. ABCD Arq Bras Cir Dig 2010;23(3):183-186.

32. Grittem L, Méier MJ, Gaievicz AP. Visita pré-operatória de enfermagem: percepções dos enfermeiros de um hospital de ensino. Cogitare Enferm. 2006;11(3):245-51.

33. Lopes CMM, Galvão CM. Posicionamento cirúrgico: evidências para o cuidado de enfermagem. Rev. Latino-Am. Enfermagem. 2010;18(2):[08 telas].

34. Silva FG, Oliveira Junior NJ, Oliveira DO, Nicoletti DR, Comin E. Análise de eventos adversos em um centro cirúrgico ambulatorial. Rev SOBECC. 2015;20(4):202-9.

35. Caldana G, Gabriel CS, Bernardes A, Évora YDM. Indicadores de desempenho em serviço de enfermagem hospitalar: uma revisão integrativa. Rev RENE. 2011;12(1):189-97. 